NCBI Bookshelf. A service of the National Library of Medicine, National Institutes of Health.

Haggett A. A History of Male Psychological Disorders in Britain, 1945-1980. Basingstoke (UK): Palgrave Macmillan; 2015.

\title{
Chapter 5 Special Cases: Sick Doctors and Ethnic Presentations of Psychological Illness
}

\section{Introduction}

By the 1980s, two particular concerns had begun to catch the attention of those interested in mental health. The first was the realisation that medical professionals (and GPs in particular) appeared to be particularly vulnerable to mental ill health and addiction to drugs and alcohol. The second was a growing concern about the psychological health of those who had emigrated to Britain in the decades following the Second World War. They are explored here because together they are illustrative of many of the broad themes already explored in this book, and serve to advance the core arguments put forward in earlier chapters. Concerns, for example, surrounded the working practice of doctors and the provision of support should they require it. Alcohol consumption among doctors, too, heavily influenced the approaches taken towards patients who presented with possible alcohol addiction. Among ethnic minorities, discussions explored sickness absence and absenteeism, reflecting many of the debates explored in Chapter 2. Among both groups, in different ways, the ability (or otherwise) to recognise psychological illness and the willingness to report it further elucidate our knowledge of male psychological illness. Although their experiences are very different, their stories bring together much of what has been revealed thus far.

\section{Sick doctors}

In an influential article that appeared in the British Journal of Psychiatry in 1967, M. F. a'Brook and two colleagues, J. D. Hailstone and I. E. J. McLauchlan, undertook a study of physicians receiving in-patient care for psychiatric illness at two hospitals: St Andrew's, Northampton and Atkinson Morley's Hospital, Wimbledon. Given the potentially serious implications of psychiatric illness in medical professionals, the authors expressed their surprise at the dearth of research on the topic. Only two published studies existed on the subject, and these were American. No British research existed, as far as they could discover. ${ }^{-}$The authors found no statistically significant difference in levels of psychiatric disorder between the in-patient group and a control group of doctors - a finding that was to be disputed by further research in later years. The study nonetheless confirmed that there was a serious problem with drug addiction and alcoholism within the medical profession - a finding that had been causing concern for some time. The authors also acknowledged 'the difficulty in differentiating functional somatic symptoms from organic ones in a medically sophisticated patient', and suggested that doctors may well become addicted to alcohol or drugs 'either as a consequence of neurotic symptoms or as a defence against their development'. ${ }^{2}$ Some ten years later, Robin Murray, who wrote widely about psychiatric illness in the medical profession, claimed that a'Brook's figures wildly underestimated the gravity and extent of the problem. Murray's investigation into admissions and discharges from Scottish mental hospitals and psychiatric units indicated that doctors were significantly more likely to experience depressive disorders and psychosis as well as drug dependence and alcoholism. Murray was keen to point out that his research had taken into account the size of the population from which the sample had been drawn, making his study more reliable than previous surveys.

Concern about the use of alcohol and drugs among doctors can be traced to the early 1950s. Max Glatt, who featured regularly in debates about alcohol (see Chapter 3), noted that his interest in the problem had been aroused during the early 1950s when studying the contribution made by alcoholism to drunken driving. Many of those who had been admitted to his alcoholic treatment unit at Warlingham Park Hospital were doctors, and over 50 per cent of them had admitted to having been 'in trouble with the law through drunken driving,. ${ }^{4}$ Many of those Glatt treated continued to take risks driving whilst under the influence, even after serious accidents. This situation led him to believe that repeated driving in an alcohol-impaired state was 'a common prodromal symptom in alcoholism,. Glatt noted that doctors were greatly over-represented in samples taken from alcoholic populations and supported this contention by providing statistics of liver cirrhosis mortality from the Registrar General which suggested that rates of death were three-and-a half times greater among doctors than among the general population. ${ }^{6}$ Drug addiction was also noted to be a significant problem among doctors. a'Brook observed in his study during the late 1960s that barbiturates and amphetamines were the most commonly used drugs. ${ }^{7}$ Murray found that the rates for alcoholism and drug dependence were respectively 2.6 and 5.3 higher among doctors than the general population. 
On both sides of the Atlantic, suicide had long been considered a concern among members of the medical profession. An editorial in the British Medical Journal in 1964 pointed out that high suicide rates among doctors had been recorded since the early decades of the twentieth century. Initial interest in the US had indeed been incited by a cable dispatch from London in 1903 announcing a great increase of suicides among physicians in Great Britain. ${ }^{9}$ Between 1949 and 1953, there were sixty-one suicides among male doctors aged between 25 and 64 in England and Wales, and another thirteen among older doctors. 10 The editorial cautioned that these figures were still on the conservative side since reports based on death certification underestimated the true extent of the problem because many cases were not declared as suicide. In a letter to the $B M J$ much later in 1989, a'Brook pointed out that the incidence of suicide in the US had decreased quite significantly by the 1980s due to the development of sick doctor programmes throughout the country. By the 1980s in Britain, in contrast, the incidence of doctors committing suicide was more than three times higher than that for the general population. ${ }^{12}$ The overwhelming consensus among researchers was that one of the principal reasons for such high rates was the availability of poisonous drugs. Almost all doctors who killed themselves used drugs and, not only did they have access to them, but also held the required toxicological knowledge. ${ }^{13}$ A number of researchers also observed that the medical speciality was an influential feature since a disproportionate number of cases appeared to come from psychiatry. ${ }_{15}^{14}$ GPs were also thought to be particularly vulnerable to psychiatric disorder and addiction.

Etiological explanations about mental illness, addiction and suicide in doctors were broadly formulated around two opposing camps: one that identified the unique aspects of life working in medicine as the cause; the other proposing that medicine might attract those with personality traits that made them inherently vulnerable to mental illness. A number of commentators suggested that many of the personality traits which characterised a good doctor might predispose him to depression. ${ }^{16}$ Others suggested that psychiatry as a speciality may attract more doctors themselves in need of psychiatric help. Murray, for example, while not discounting environmental factors, noted that many alcoholic doctors also had personality disorders. ${ }^{18}$ Glatt, in contrast, and in line with his general approach towards alcoholism, stressed the importance of environmental factors, suggesting that the continual excessive emotional and physical demands of medicine might prompt doctors to self-medicate with drugs and alcohol. ${ }^{19}$ Some studies suggested that physicians were appreciably more 'anxious' and that this might be directly related to fears of inadequacy in fulfilling the professional role. ${ }^{20}$ Others indicated that psychiatric illness in physicians was a vulnerability that existed prior to entry into university and that individuals with an obsessive personality type were attracted to medical school. ${ }^{21}$ The age-old dichotomy between environmental and individual causes was, of course, never entirely disentangled, and increasingly, research suggested that both views should be taken into account. ${ }^{22}$ In Britain, evidence submitted to the Goodenough Committee in 1944 raised concerns about the failure of medical schools to 'exclude men and women who, though able to pass examinations, ha[d] not the requisite aptitude, character or staying power for a medical career'. The committee agreed that there should be machinery not only to select students from this standpoint but also to weed out students who proved unsatisfactory. However, 'no one was bold enough to state the criteria of rejection, or more specifically, to say whether a propensity to some form of psychological illness should be regarded as a sign of unsuitability'.

One aspect of the problem that attracted broad agreement was the acknowledgement that the shame and stigma surrounding mental illness and addiction affected doctors even more acutely than those outside the medical profession. All research suggested that doctors rarely sought help of their own accord, even when they were concerned, for example, about their own alcohol assumption. Many accounts indicated that medical colleagues would 'turn a blind eye', even if the situation was developing into a crisis. ${ }^{24}$ One rare and brutally honest account written by Gareth Lloyd, a physician who had become an alcoholic, is worth repeating in detail since it articulately encapsulates what must have been the situation for many alcoholic doctors. He recalled:

I began to drink alcohol for symptomatic relief and to drink earlier in the day. No one around me seemed to notice, or if they did so, nothing was said to me. Daily intake of alcohol gradually increased and with this came more symptoms, a worsening overdraft and a loss of interest in my chosen speciality. Each clinic or operating session became an increasing burden to dovetail into a demanding drinking pattern . . . by some miracle of effort I maintained good clinical standards and obtained an MRCOG.

Lloyd eventually sought help, but alternated for many years between periods of sobriety and 'falling off the wagon'. He described the situation as 'difficult for a proud man to accept. The frustration of failure, the humiliation of despair, only increased an irrational impulse to find a way to drink safely'. In publishing his own account (and maintaining a long-term interest in alcoholic doctors and their treatment), Lloyd made a plea for greater openness and 
understanding, concluding that "surely the time has come to speak more freely of the illness that dare not speak its name'. Glatt echoed Lloyd's sentiments based upon his own experience treating alcoholic doctors:

As regards alcoholism, the average doctor has not become better educated at medical school about alcoholism than the lay public, and he shares with the layman all the prevalent wrong notions, which maintain the stigma. It therefore does not dawn on the drinking doctor for a long time that he himself could possibly be an alcoholic, 'after all, he is not a psychopath, not a moral weakling, not a skidrow type' . . He may certainly feel ashamed of his inability to keep himself under better control but he cannot let others know about his 'weakness', and so he may dose himself up with barbiturates or tranquilizers - and may well become dependent on them as well. ${ }^{27}$

As a'Brook noted, when it came to psychiatric symptoms, doctors occupied a privileged position in society, which enabled them to seek advice informally from their colleagues. Some chose to consult a non-psychiatric colleague rather than a psychiatrist and might avoid or refuse referral. Those who did seek psychiatric assistance were often reluctant to admit to it later or to discuss their progress. Indeed, according to a'Brook, 'many doctors with neurotic illnesses never reach[ed] the psychiatrist'. 28 Physicians also often encountered difficulties adopting the patient's role and their psychiatrists frequently '[found] it impossible to adhere to the consistent therapeutic policies that apply to other patients'. ${ }^{29}$ The stigma of a psychiatric diagnosis was potentially very damaging to a physician's career, and as a result, some suspected a tendency among psychiatrists to diagnose 'less pathological' conditions in their medical colleagues. ${ }^{30}$ Research from in-patient units suggested that physicians often discharged themselves early and discontinued treatment against the advice of their psychiatrists. In one American investigation of the Mayo Clinic in-patient psychiatric services at Rochester Minnesota, a high number of doctors discharged themselves against medical advice, the authors noting explicitly that, 'this may be related to the difficulty the physician has in accepting his illness and his status as a patient'. They added that this situation was subsequently not without strain for hospital staff 'who must cope with their own feelings of insecurity in dealing with the physician as a patient'.

Anthony Allibone, a GP from East Anglia who was chairman of the General Medical Council's Health Committee during the early 1980s, became interested in the health of doctors and explored the subject in a chapter published in the Medical Annual in 1983. Drawing on the only available evidence of doctors' views about their health, taken from The Survey of the Health Care of Doctors (1977-1979), Allibone noted that 46 per cent of doctors surveyed had at some time delayed seeking medical help when they needed it, and nearly a third said that with hindsight they had delayed longer than was prudent. Many reported that the consequences of not seeking help had had an adverse affect on their husbands or wives. ${ }^{33}$ For over half of the respondents, the GP with whom they were registered was a personal friend and for 70 per cent of GPs, their own doctor was a colleague from the same surgery. Perhaps unsurprisingly, self-treatment was common. Although in some circumstances self-treatment was deemed by Allibone to be appropriate (in the case of treatment for a common cold, for example), a number of doctors admitted to treating their own mental illness or alcoholism, something that would be clearly contra-indicated. ${ }^{34}$ Allibone opened his chapter with an anonymous contribution from a GP who emphasised the apparent 'conspiracy to reject illness that might reflect on professional competence'. He went on to recall a distressing incident in which a doctor near him had become alcoholic and depressed and eventually shot his wife and children while working in the health centre. The GP was dismayed that the sick doctor 'was somehow unable to communicate his distress to his colleagues'. 35 By the 1980s, such calls for awareness were by no means exceptional and other doctors wrote in to the medical press expressing alarm at the situation. A student midwife, for example, wrote to the correspondence section of the $B M J$ in 1983 complaining that she had recently witnessed one of her colleagues - a doctor - 'break down'. Although, as his friend and colleague, she had often discussed with him the stresses of the job and its consequent effects, the student articulated a great sense of guilt and shame that, even though she had recognised he was depressed, she did nothing as there was no one to whom she felt she could turn: 'Who would listen? Who would care?',

Allibone, in the same correspondence section of the journal, put forward criticism of the framework underpinning the GMC's health committee and the general view that it had been bound 'hand and foot' by an inability to integrate successfully the obligation to 'care' along-side a judicial role. ${ }^{37}$ Concerns about the ways in which sick doctors were disciplined had emerged during the Committee of Inquiry into the Regulation of the Medical Committee, under the chairmanship of the British physicist Alexander Walter Merrison, reporting in 1975. The Medical Act of 1978 which followed, although primarily concerned with the broader regulatory aspects of the medical profession and medical education, also separated disciplinary processes from those that dealt with doctors whose performance was impaired by ill health. ${ }^{38}$ In practice, however, the health committee that was designed to protect the rights of the doctor was still entirely unsatisfactory. The system failed to cope with the alcoholic doctor and 'was more concerned with his inability 
to provide a service than with his fitness to practice'. The GMC's submission to the Merrison Committee had revealed that at least half of the doctors appearing before the council on disciplinary charges were suffering from the effects of alcohol misuse, drug abuse or mental illness. ${ }^{40}$ However, until the medical committee was set up in 1985, the council could take only disciplinary action against a doctor and was powerless to prevent a doctor from practising unless the issue was one of serious professional misconduct. It often showed a tendency to postpone judgement because of a reluctance to strike a mentally ill doctor off the register. The GMC thus, in part, colluded with the chronic alcoholic doctor in allowing him to continue in practice. ${ }^{41}$ It is striking that, over thirty years later, physicians within the NHS still report high rates of psychological distress: depression, substance abuse, alcoholism and suicide, leading commentators more recently to describe a 'disturbing view' of the caring profession and the approach of the GMC as 'one of disinterest, which is temporarily discarded when disaster overtakes'.

Not only was the regulatory framework of the GMC not conducive to exposing and supporting doctors with mental illness and addiction problems, but there was also an uneasy acknowledgement that students at medical school habitually drank heavily as part of an accepted culture before they qualified. Studies began to suggest that a pattern of heavy drinking often began at university and became entrenched during professional life, sometimes leading to a breakdown, on average fifteen to seventeen years into medical practice. ${ }^{43}$ Glatt expressed explicit concern about the level of alcohol consumption at university, and, in his work on alcoholic doctors, included an anonymous contribution from one physician who recalled that he was always regarded as the odd man out in medical school because he could not down three pints in a lunch hour. ${ }^{44}$ Robin Murray echoed these concerns and cautioned that 'an ability to hold one's liquor is said to be almost mandatory for medical students. The majority of them enjoy trying to measure up to this caricature, but for an unfortunate few, heavy drinking as undergraduates or housemen may be the prelude to later alcoholism. ${ }^{45}$ As Glatt pointed out, alcohol experts had for some time suggested that in nations or groups with high social acceptance of heavy drinking, even average, emotionally stable personalities may expose themselves by habitual heavy social drinking to the risk of becoming (in time) dependent on alcohol. According to Glatt, therefore, it was perhaps not surprising that doctors, who as medical students may often have come to regard occasional heavy drinking as nothing extraordinary, later in life may continue this habit. ${ }^{46}$ A further consequence was that a culture of heavy drinking understandably blurred some physicians' appraisal of what was normal or abnormal drinking among their patients. A number of the doctors interviewed for this project confirmed that heavy drinking was an accepted part of medical school. The recollections of David Palmer were typical of many:

There was a complication in medicine that in fact, medical schools were just awash with alcohol. And these young men drank. They were, in my generation, 85 per cent were male . . . And I have no doubt at all . . that they were drinking, for bravado, to escape the emotional stress of what was happening to them, and it was a kind of escapism. And they drank ludicrously. And of course what happened was that doctors came out of medical school, my generation anyway, almost thinking that heavy drinking was pretty normal.

Some doctors had colleagues who had succumbed to drink or drug addiction in later life. One GP remembered a friend and fellow physician who became a pethidine addict, in his view due to the stress of the job and the availability of drugs. On alcohol, he remarked, laughing, that the standard joke of the time was: 'What's the definition of an alcoholic? Somebody who drinks more than their doctor. ${ }^{48}$ A study of drug abuse among medical students at Glasgow University in 1971 suggested that, although the problem was small, it was more common in men and that drug use was more likely in those who drank alcohol regularly.

There were inevitably negative consequences for the wives and families of doctors afflicted by mental illness or addiction. Increasingly, commentators from within the medical profession and families themselves began to draw attention to the strain placed on family members. Echoing the findings discussed in Chapter 2 of this book about family presentations of illness, an American study in 1965 showed that it was not uncommon for doctors' wives to present with psychiatric symptoms around the time that their husbands 'broke down'. Many of the participants blamed the cause of their symptoms on relationship difficulties caused by the increasing exclusion from the husband's life as he became more and more involved in his profession. ${ }^{50}$ Many of these wives were addicted to drugs such as morphine or morphine-derivatives, prompting the author of the study to conclude that the addiction was related 'dynamically and empirically to the profession of the husbands'. 'A review article on the subject of psychiatric illness in the medical profession covering research on both sides of the Atlantic reported that marital discord might precipitate or result from psychiatric illness in doctors. Divorce was, perhaps unsurprisingly, twenty times more common among British doctors hospitalised for psychiatric disorders. ${ }^{52}$ Echoing the earlier American study, this overview of existing research reported that doctors' wives most usually became ill during their thirties although their 
illness might well have been present for six or more years. Drug and alcohol abuse were common, as were complaints about sexual relations, thoughts about suicide and somatic disturbances. Although the tone of this article indicated that the expectations and demands of the physician's role were the most likely cause of such problems, some still suggested that the personalities of husband and wife may play a part, particularly where 'a dependent histrionic woman with an intolerable need for affection and nurturing' is attracted to a physician who becomes detached, aloof and a compulsive worker. ${ }^{33}$ In contrast, others suggested that wives and families played an important role in helping physicians face up to their problems and were often the ones to apply pressure on them to seek psychiatric help. ${ }^{54}$ Britain, by the 1980s, doctors' wives indeed played an important role in campaigning for less damaging working practices. The wife of a senior GP, Jill Pereira Gray, drew attention to many of the problems facing medical families and the ways in which they were vulnerable to the particular strains associated with the professional medical role. Speaking openly about the topic, she argued, would ensure that the subject of the doctor's family could move, as it rightly should, 'from the shadows to the stage'. ${ }^{55}$ Such publicity and pressure lead Allibone to note by 1983 that, as a consequence, there was 'no doubt about changing attitudes which "may profoundly influence doctors" expectations of medical care for themselves and their families'.

Those who raised concerns about mental illness and addiction in medical professionals put forward three broad recommendations to help sick doctors and prevent them being vulnerable to it in the first place. Firstly, there was overall agreement that more emphasis should be placed at medical school on preventing habitual alcohol consumption and awareness of its dangers. Glatt, for example, argued that doctors should be specifically targeted as a 'high risk' group. Special education at undergraduate level, he suggested, would raise awareness that doctors might be vulnerable to alcoholism on two counts: the temptation of relief drinking and the acceptance of heavy drinking by those around them. Raised awareness would ensure that doctors would not only be less likely to become a casualty themselves, but also '[they] would be in a position to suspect the development of alcoholism early on in [their] patient's drinking career and to arrive at an earlier diagnosis.' 58 Glatt warned that 'the outcome of the still-prevailing laissez-faire attitude to education and the early diagnosis and treatment of alcoholic doctors will be many more avoidable cases of dead doctors and perhaps dead patients. ${ }^{59}$ Others maintained that standards of teaching in psychiatry should be improved at both undergraduate and postgraduate level and suggested that there should be better liaison between psychiatrists and members of other branches of the profession.

Recommendations for special help-groups for doctors, such as the British Doctors' Group, were also put forward. This organisation originated in 1973, when two medical practitioners who were experiencing difficulties with alcohol abuse met up to discuss their difficulties. They discovered that they were able to relate to each other's problems, some of which were unique to life in the medical profession. The group soon took on new members, including female doctors, dentists and doctors addicted to drugs. The meetings were in addition to attendance at AA. ${ }^{61}$ Glatt spoke highly of this organisation, describing it as 'one of the most hopeful developments in this field in the country', and maintained that doctors often recovered well within appropriate therapeutic communities. ${ }^{62}$ Other schemes eventually developed within specialisms, such as those arranged by the Society of Anaesthetists and the Royal College of Psychiatrists 'to provide rapid, confidential and informal help for the colleague suffering from mental ill health, alcoholism or drug abuse'. The Norfolk Medical Care Scheme was also held as a good example of what was possible. In this scheme, developed by the Norfolk Local Medical Committee, with the support of local members of the Royal College of General Practitioners, a doctor would be identified as a 'link' between the sick doctor and the general practitioner caring for him. ${ }^{64}$ In addition to these recommendations, some maintained that medical school admission departments should ensure that well-rounded individuals were selected, 'whose academic achievements complement rather than substitute for a stable personality'.

Finally, when it came to the cause of mental illness in doctors, the ethos of the medical model and the structure of medical training did not entirely escape criticism - particularly in the US. Samuel Corson, professor of psychiatry and biophysics at Ohio State University (who later became known for his work on pet-assisted therapy), wrote an article in 1981 with his wife Elizabeth (who was his laboratory assistant) addressing aspects of social stress in medical education. Applying a biopsychosocial and systems theory approach, the Corsons expressed deep concern that 'physicians have a suicide rate twice that of the population they are trying to keep healthy'. Although fully accepting that psychological stamina was of vital importance to a medical student if they were to become a sound physician, they were critical that so little attention had been focused upon patterns of medical training that may contribute to doctors' morbidity and mortality. ${ }^{66}$ The Corsons suggested that it was entirely possible that the highly regimented, stress-inducing methods of medical training contributed to the 'dehumanising' of doctors, driving some of them to addiction and suicide. ${ }^{67}$ The authors cited a number of alarming personal accounts from junior doctors who described 
long hours, sleep deprivation and unreasonable workloads. Added to the mechanistic, dehumanised approach fostered in medical training, the Corsons argued that these factors cumulatively 'mitigate[d] against the ability to learn or to develop attitudes of compassion and caring'. Medical education was thus 'based on dualistic concepts, with the physician being concerned primarily with treating the body as though human beings are inanimate objects, not subject to psychological and emotional influences'. ${ }^{69}$ Their conclusions were unequivocal and largely accord with the oral history testimonies of GPs and the broader themes that emerged from Chapter 1 of this book. Firstly, they suggested that the prevailing reductionist medical model had a tendency to 'weed out the most sensitive, creative and humanistic physicians'. Secondly, they argued that, for those who remained in training, the medical model tended to develop a cynical, callous attitude and insensitivity to human needs and suffering. Thirdly, their view was that medical education fostered a competitive atmosphere that might not be fitting or conducive to the caring role. Finally, the authors concluded that the unintended consequences of this model might be the enhanced the risk of iatrogenic errors. They cautioned more broadly that 'the type of physician we train will have the major influence on the kind of health care we will get, including the health of those whose mission it is to provide health care' 70

\section{Ethnic presentations of psychological illness}

Symptoms of psychological and psychosomatic illness in immigrant communities have long been a source of interest and concern, not only for the medical profession, but also for sociologists, anthropologists, politicians and historians working on the impact of migration. A full analysis is beyond the scope of this book and there is much more important work to be done, particularly with respect to historical work. Nonetheless, where this study touched upon urban communities, the health and welfare obstacles faced by immigrants who had arrived in Britain - and the challenges presented to doctors responsible for helping them - emerged as important themes.

Immigration trends over the twentieth century are well known. Prior to the period under study, the largest migration population in Britain was the Irish. During the period between 1800 and 1914 approximately one million people crossed the Irish Sea to settle in Britain. ${ }^{71}$ Although on a smaller scale, the Jews, eastern Europeans, and communities of people from western Europe also journeyed to Britain. Significant numbers of non-Europeans did not arrive until after the Second World War, since when large numbers have migrated from the Caribbean, South Asia, Hong Kong and Africa - while smaller numbers have moved from the Americas. ${ }^{72}$ Immigration from the continent has also remained constant, with large numbers of people arriving from Ireland, Poland and Italy - and, in recent years, also from other eastern European states following the accession of new members to the European Union. Post-war, immigrants increasingly settled outside of the traditional communities in London, to the Midlands and other cities.

Not only has immigration changed the demography and economic development of Britain, but also, as numerous commentators have noted, it has radically changed concepts of identity and 'Britishness'. 74 From the 1960s, there was increasing anxiety about the health and well-being of immigrants; however, there were few scientific investigations on the subject due to the fact that 'the study of ethnic differences in patterns of disease . . often spilled over into political and philosophical areas, stifling objective investigation and rational discussion'. ${ }^{75}$ Commentators writing during the 1970s noted that the topic was 'fraught with issues of political, economic and social concern, since understanding and sympathy are not too frequently shown to the migrating individual or group by the receiving society'. ${ }^{76}$ With particular reference to mental illness, some have cautioned more recently, that 'to discuss the psychological adjustment of ethnic minorities is to underline yet again the popular conception of them as being primarily a problem ${ }^{77}$ There were a small number of investigations undertaken during the 1930s and 1950s on migration and mental illness; however, post-war, political and cultural sensitivities largely forestalled rational discussion about the ways in which immigrant communities coped with the social and cultural pressures of settling in an unfamiliar environment.

Broader international concerns about how psychiatric illness might present differently in non-western populations had become the focus of study during the mid-1950s in Canada when Eric Wittkower, who later came to work at the Tavistock Clinic in London, established a programme of 'transcultural psychiatry' at McGill University. The movement that developed from the ensuing collaboration between psychiatry and anthropology sought to provide a framework for integrating knowledge in different parts of the world and to provide an institutional core within which international programmes could be harmonised. ${ }^{80}$ Transcultural psychiatry, however, soon found itself at odds with the increasingly reductionist biomedical model promoted by psychiatry, which assumed the universality of mental illness. Psychiatry's position opposed the notion put forward by transcultural psychiatry that 'emphasised the importance of understanding disease in the terms of the patient's culture within the framework of cultural relativism' ${ }^{81}$ In Britain, the movement's research focused primarily on immigrants and racism within psychiatry, 
chiefly the notion that members of ethnic minorities were 'preferentially psychiatrised'. . Its stated aims were thus to 'promote the equality of mental health irrespective of race, gender or culture'; and, as recent authors have pointed out, although the term 'culture' was retained, it was primarily the impact of racism that became the focus of the organisation. Indeed, the first book on the subject to be published in Britain, by Bradford psychiatrist Philip Rack, entitled Race, Culture and Mental Disorder, was not published until 1982.

Immigration was (and is) of course a complex phenomenon. The decision to emigrate might be deliberate or involuntary - forced by conflict or economic exigency. Movement might be overseas, internationally inland or internally within one country. Researchers noted that immigrant communities experienced pressures that were usually dependent upon two factors: the cultural background of the immigrant and the socioeconomic and cultural characteristics of the community into which they arrive. With the transcultural psychiatry movement in Britain still in its infancy during the 1970s, major environmental change was the defining aspect of migration that inspired interest among the small number of existing researchers. These individuals hoped that the study of mental disorders in a migrant population would offer good opportunities to gain knowledge about the causes of mental illness more generally.

In accord with broader discussions about the causes of mental illness, those who were interested in ethnic presentations and immigrant communities tended to align themselves on one side of the familiar debate about the relative influences of constitution and environment. On the one hand, statistics sometimes supported the "negative selection' hypothesis that suggests individuals who develop mental illness might be more likely to migrate in the first place. Ødegaard's early study of Norwegian-born immigrants and native-born Americans in Minnesota, for example, found high rates of schizophrenia among Norwegian immigrants and migrants who then returned to Norway. Ødegaard explained this by suggesting a greater tendency for ' pre-schizophrenic individuals to migrate' ${ }^{86}$ A. G. Mezey's 1960 study of psychiatric illness and migration also suggested that personality factors played an important role in bringing about the migration of certain individuals in the first place and, therefore, 'probably underlie[d] the high incidence of schizophrenic disorders in migrants. 87 Age also emerged as an important factor. Many studies revealed that there was an excess of adolescent and young adult schizophrenia among migrants; however, serious psychotic illness tended to appear more regularly in this age group more generally, regardless of ethnic origin. Sex and class were considered to be additional influencing factors. Among hospital admissions was a preponderance of young males, but the fact that young males seeking work were often the ones to emigrate might again explain this factor. ${ }^{88}$ Married persons appeared to have lower hospitalisation rates than single people and rates were much greater for the lower than for the upper and middle classes. ${ }^{89}$ Hospitalisation rates for specific ethnic groups tended to be inconclusive, although American studies noted that 'rates for Negroes [w]ere usually much higher than rates for whites'. In general, authors maintained that 'the foreign-born had higher mental hospitalisation rates than nativeborn regardless of cultural or ethnic origin'; ${ }^{91}$ however, as this chapter will demonstrate, the way in which individuals presented with illness varied widely between different cultural groups.

The environmental stresses of migration were nonetheless also considered to be important. The way in which an individual had prepared for the change and his $_{92}$ or her general state of health prior to migrating were seen as important factors in the development of mental illness. ${ }^{92}$ The attitudes of those in the new community and the availability or otherwise of social support networks were also viewed as paramount. In Britain, immigrants from the New Commonwealth and Pakistan tended to settle in inner-city areas such as Tower Hamlets, Lambeth and Islington in London where the housing shortage was already acute. With the exception of those employed in the medical profession, the jobs taken by immigrants were often characterised by insecurity and low wages, and many lived in overcrowded housing with poor amenities. ${ }^{93}$ Recent scholarship, drawn from the Community Relations Commission in 1977 and the national census of 1971, has confirmed that many immigrants experienced significant disadvantage in housing, unemployment and family life. ${ }^{94}$ In addition to these factors, reports from the 1970s indicate that immigrants endured a range of discriminatory practices in recruitment for jobs and by private landlords.

Most commentators were unable to conclude whether constitutional or environmental factors were responsible for the high rates of mental illness among immigrants and increasingly accepted that there might be a multiplicity of explanations. Existing studies were drawn from hospital in-patient data and dealt only with serious psychotic illness. Very little was known about the less severe affective disorders that remained undiagnosed in the community; however, as we shall see, oral history testimonies from GPs who worked in inner-city communities illuminate some of the problems faced by immigrant communities. Occupational health surveys also indicated distinct patterns of sickness and absenteeism between groups. Early international studies suggested that immigrant workers were absent from work more frequently than indigenous employees, but very little research existed on the subject in Britain. ${ }^{96}$ The first 
significant study at home focused on a large manufacturing company in south-east England. The authors began by explicitly stating that research on mental health and race had hitherto been inhibited by political and cultural sensitivities. The survey found that Asian employees had considerably more sickness absence in all categories. They had more individual spells of sickness and fewer employees in the 'no certified absence' group. On average, Asian workers had twice as many days off work as Caucasians. However, most absences were of short duration, unlike Caucasians and West Indians who were more likely to take longer spells off work. ${ }^{98}$ The authors drew a range of inferences from the project and acknowledged that there were a number of non-cultural factors that should be taken into account - first and foremost, much of the documented absenteeism involved younger workers, and this was also a consistent finding among white employees and in other occupational health studies. Immigrant workers nonetheless often endured accommodation problems and were more prone to ill health due to poor living conditions and poverty; however, the authors noted that this would apply to other non-Asian immigrants and could not therefore explain why Asians predominated in figures for sickness absence. A number of culture-specific factors were noted. The English language, for example, was the national language among West Indians, but Asians spoke it less well. Communication problems might reasonably cause integration obstacles, stresses and strains leading to ill health and absenteeism. Drawing on previous studies on pain thresholds, the authors also suggested that cultural sensitivities towards pain and illness provided an alternative explanation for pronounced variation in sickness absence. Pain from muscular strain or arthritis - or pain with a psychological origin - was thought to be experienced differently by groups with different cultural backgrounds and might explain much of the documented sickness absence.

Research on ethnic presentations of psychological illness in general practice was even more limited. Stuart Carne, a London GP working in Hammersmith, commented on the difficulties investigating such a sensitive topic, noting that the very word 'immigrant' was liable to trigger emotive reactions since it was used by some as a term of abuse. Over half of the patients on Carne's list originated outside Britain and he found a range of physical complaints that were more commonly seen in those with non-British nativity. Raised blood pressure was 'a known hazard' in west African patients, while peptic ulceration appeared to be more common in West Indians. ${ }^{101}$ Immigrant patients, particularly females, were noted to attend the doctor's surgery more frequently, but required fewer home visits. When compared to British patients, they received less prescribed medication, but were issued sickness certificates more frequently (perhaps in accordance with the findings from occupational health studies). ${ }^{102}$ Carne scarcely mentioned psychosomatic presentations of illness, except to say that 'headaches of a non-specific type' were very common in immigrants. However, a hospital physician from Birmingham, Farrukh Hashmi, drew attention to the problems of adaptation endured by immigrants, which invariably caused aches and pains, hypochondriasis and psychosomatic diseases, or 'other signs of anxiety and neurosis'. 103 In a paper on emotions and adaptation published in 1970, Hashmi described many of the cultural presentations that were to become the focus of attention for Arthur Kleinman in his influential work on somatisation some years later. Hashmi observed that, for example, when depressed, Pakistani men often complained of sexual weakness and nocturnal emissions due to the fact that in the East, and in the Pakistani patriarchal society, the father is the dominant figure in the home and a great deal of mystique existed about manhood and sexual potency. ${ }^{104}$ In contrast, West Indian men tended to present with physical aches and pains connected to their particular cultural construction of 'manliness', which emphasised the importance of physical strength. Hashmi cautioned that these presentations were usually related to the cultural, social and religious upbringing of the patient and that it was imperative that physicians understood the cultural influences that shaped ethnic presentations of stress and psychological breakdown.

If recognising and treating complex psychological and psychosomatic symptoms in British men within the prevailing western medical model was not problematic enough, GPs working in areas populated with large numbers of immigrants were faced with considerable additional challenges. Carne noted that language difficulties created a communication barrier and that sometimes patients who appeared to be 'speaking the same language use[d] words differently,. ${ }^{106}$ Further, he argued that patients tended to come to the doctor with preconceived ideas about what was wrong with them and what was likely to happen at the consultation. For immigrants, previous medical experiences were usually very different to those of English patients who had twenty years of experience of treatment under the NHS. James Robertson, a GP who had spent his whole medical career working in the East End of London, pointed out that 'first generation' Bangladeshi female immigrants spoke poor English and rarely left the home. Often, communication would be through one of the children, typically 'a twelve year old boy, because it was the boy who came out, because you needed a male member of the family to accompany you . . . it had to be your son. So very often it was the sons translating for the mothers'. 108 In areas with high levels of poverty, Robertson explained that comorbidity was a real challenge. Mental illness and serious physical conditions such as lung disease, heart disease and diabetes often existed together and this 'made life very hard' for patients, and difficult for the doctor trying to 
'separate out' the dual diagnoses. ${ }^{\text {IVy }}$ On his list were large numbers of older men from Somalia, Ireland and Scotland who lived in local hostels. These men, according to Robertson, were often unmarried and socially isolated. 'Major depression' was common among them. ${ }^{110}$ Sarah Hall, another GP who worked for many years in the East End of London, recalled that the male Bengali population was also socially isolated, with no support system. Their English was very poor and they had no advocacy interpreters. She noted that 'you wouldn't really know at all what was going on with them'. $M e n t a l$ and physical diseases were often exacerbated by addiction to alcohol and drugs. Heroin addiction became a 'devastating' problem in the East End of London during the 1980s. ${ }^{112}$ Compliance with medication regimes also differed between groups. Bangladeshis were in general very compliant with prescriptions and medication, whereas Afro-Caribbean patients were less keen on taking medicines or relying on traditional western medicine.

Psychosomatic presentations were common in both men and women from different ethnic backgrounds; however, Sarah Hall maintained that for women there would 'be a much more rapid shift into a psychiatric domain'. ${ }^{114}$ Both GPs with experience treating immigrant communities were of the opinion that patients who somatised were not able to express distress beyond bodily pain. Robertson maintained that if one were to ask any east London GP, they would tell you how difficult it was to manage psychological symptoms in immigrant groups because of what he described as the 'I hurt all over' syndrome. ${ }^{115}$ As Hashmi had noted in his paper in 1970, presentations were often culturally specific. Hall suggested, for example, that a psychological diagnosis would be seen as threatening in Bengali culture because it would suggest weakness:

If the psychological domain meant you were weak, that you might have a family weakness ... that might be very troublesome when your daughters or sons came to get married, an alliance, you know. So any hint of weakness was really quite difficult.

Hall explained that, while wishing to avoid generalisations about all Bengalis, most often, as patients, their favoured discourse was in the physical domain, articulated through some kind of pain - usually gastrointestinal or musculoskeletal. Often a patient would present with a long list of different pains and would be reluctant to accept a psychological diagnosis due to the stigma attached to it. According to Hall, for example, the Bengalis did not have a word for 'depression' in their culture. ${ }^{\text {} 17}$ Often, patients would be uncomfortable with the language and the concepts of western medicine. Eventually, Hall realised that it was mostly counter-productive to apply western concepts and illustrated this with an anecdote about a Bengali man who came to her surgery complaining of waking up paralysed, feeling as though he was being strangled. His concern was that somebody had put 'a jinn' on him, and he had asked the imam to put some incense and amulets around the room. After some discussion, it emerged that the patient had been feeling 'low' and that his wife had left him. Hall attempted to explain that he might be experiencing a condition known in western medicine as a hypnopompic hallucination whereby a person can wake up feeling paralysed. They agreed ultimately that they both had their own 'understanding' about what was happening and the patient ended up needing no further intervention. Hall described this as an 'intercultural encounter' and stressed the importance of what she called 'culture brokers' or health advocates who can help with consultations and understand the patient's culture.

In seeking to help immigrants and those with non-British back-grounds, commentators up until the 1970s had little to offer. Broad recommendations acknowledged that the British needed 'to cultivate tolerance' of immigrant groups and their cultural background. ${ }^{120}$ It was generally accepted that housing accommodation should be improved and that local authorities should examine their allocation arrangements. Other recommendations focused on concerns about physical disease and the importance of screening immigrants for infectious diseases on arrival. ${ }^{121}$ The notion that patients from non-western backgrounds might present with somatic or physical complaints which were viewed as more acceptable and less stigmatised was not formally articulated until Kleinman's study in 1977, and later developed by Laurence Kirmayer and others. Those from within the transcultural psychiatry movement were indeed later to maintain that: 'Somatisation represents a powerful method of coping with psychological distress. Symptoms are communications of distress, and in many cultures, depression connotes weakness, moral culpability and loss of face. ${ }^{122}$ Although some doctors, like Hall (who continued practising through the 1980s), developed their own skills for navigating complex presentations of disease, during the 1960s and 1970s there was little guidance or research available to aid medical professionals. When the transcultural psychiatry movement developed in Britain, its focus was primarily upon responding to racism within the profession, and not on the 'phenomenological descriptions ${ }^{, 23}$ or 'cultural explanations' for disorders. Most doctors applied a western psychiatric framework and 'superimpose[d] 
those cultural categories, ${ }^{1<4}$ upon their patients. The result was that many symptoms were excluded from a psychological domain and potential psychosocial causes underplayed.

\section{Reflections}

The more recent histories of these two groups suggest that many complex factors continue to obfuscate the detection, diagnosis and treatment of psychiatric disorders and addiction in doctors and ethnic minorities. The British Medical Association has provided extensive support services for medical professionals who experience mental illness or addiction and there are additional services available to help those who face a hearing with the GMC. Independent organisations, such as the Sick Doctors' Trust, exist to benefit those with addictions to drugs and alcohol. Nevertheless, the Department of Health's recent document, Invisible Patients: Report of the Working Group on the Health of Health Professionals (2010), indicates that a significant problem still exists. This report, which aimed to establish a framework for all healthcare organisations to build healthy workplaces, highlighted a range of ongoing problems related to the well-being of health professionals. It acknowledged that there were still higher rates of depression, anxiety and substance abuse in health professionals than in other groups of workers, noting that the work environment was often inherently more challenging and that workloads were high. ${ }^{125}$ One study cited as evidence in the report suggested that 7 per cent of GPs used alcohol frequently 'to cope', and a further NHS Trust survey found that over 60 per cent of junior doctors exceeded the recommended safe alcohol limits. One in ten of these were drinking at hazardous levels. ${ }^{126}$ Invisible Patients notes that 'suicide rates among doctors are the highest of any health professional group and are more than twice those of the general population'. 'It is striking how much of the report mirrors the concerns put forward some fifty years ago. Existing research on mental ill health of those working in the medical profession, for instance, was described as 'of limited scope and quality', and despite a 'change in attitudes', stigma was still viewed as a 'powerful deterrent' to seeking help. Informal consulting and self-prescribing were still popular: in the words of one contributor, doctors with mental health problems 'are poorly managed and under managed, and either self-prescribing or getting [their] mate to do it in the corridor'. identified as a growing problem in the NHS. The term presenteeism, coined in recent years by economists, denotes the loss of productivity caused by workers who are present at work but unwell. ${ }^{129}$ The Invisible Patients report explicitly notes that: 'Presenteeism among staff with mental health problems is thought to cost 1.5 times the amount of working time lost through absenteeism,' and cautions that the fear of repercussions increases the likelihood that staff will present at work in poor health. ${ }^{130}$ There is nonetheless evidence that some medical and dental schools have begun to formulate educational programmes that foster greater empathy and personal insight, and that in these schools, applicants are selected for personal attributes that are desirable for a caring role.

Among black and minority ethnic (BME) communities, research continues to indicate that different ethnic groups have different rates and experiences of mental health problems. The British charity, Mental Health Foundation, has found that black and minority ethnic groups are more likely to be diagnosed with mental illness and more likely to be admitted to hospital. They are also more at risk of experiencing poor treatment outcomes and are prone to disengage from mainstream mental health services, leading to social exclusion and deterioration in mental health. ${ }^{132}$ Numerous other reports suggest that BME communities are poorly served by mental health services and that individuals are reluctant to use existing services because they are not usually culturally sensitive to their needs. ${ }^{133}$ Treatment and supportive services are often based upon inaccurate assumptions and stereotypes, such as 'aggressive black men', as policy-makers and service providers fail to understand the cultural and social circumstances of BME communities and their consequent reluctance to seek help. ${ }^{134}$ Other surveys suggest that racism is widespread among BME people with mental illness and that many of those affected feel unable to speak out about their mental health. As a consequence, ${ }_{135}$ many people experience problems seeking employment, making friends and undertaking basic, everyday activities. Reflecting upon the previous fifty years, it is interesting that research continues to suggest that, although many of those from BME communities with common mental disorders are very likely to have recently seen their GP, they are less likely to have been treated for their psychological problem. A study of mental health care among ethnic minorities in 2008 suggested that: 'Many GPs fail to recognise psychological symptoms in ethnic minorities,' but also that: 'Some minority groups are less likely to present their psychological problems to GPs because they do not consider them to be the most appropriate person to treat them.' 136

\section{Footnotes}

1 a'Brook MF, Hailstone JD, McLauchlan EJ. Psychiatric illness in the medical profession. British Journal of Psychiatry. 1967;113:10131023. on 1013. [PubMed: 6060098] 
2 a'Brook, Hailstone, McLauchlan Psychiatric illness in the medical profession. 1018

3 Murray Robin M. Psychiatric illness in male doctors and controls: An analysis of Scottish hospitals in-patient data. British Journal of Psychiatry. 1977;131:1-10. on 3. [PubMed: 884403]

4 Glatt MM. Alcoholism, an occupational hazard for doctors. Journal of Alcoholism. 1976;11:85-91. on 85.

5 Glatt. Alcoholism, an occupational hazard for doctors. :85.

6 Glatt. Alcoholism, an occupational hazard for doctors. :86.

7 a'Brook, Hailstone, McLauchlan Psychiatric illness in the medical profession. :1017.

8 Murray. Psychiatric illness among male doctors. :5.

9 Editorial. Suicide among doctors. British Medical Journal. 1964 March 28;:789-790. on 789. [PMC free article: PMC1815019] [PubMed: 14097418]

10 'Suicide among doctors. :789.

11 'Suicide among doctors. :789.

12 a'Brook MF. letter to the British Medical Journal. 1989 March 4;:603. [PMC free article: PMC1835956] [PubMed: 2495136] By the late $1980 \mathrm{~s}$, it is notable that, in Britain, the rate of suicide among female doctors had reached an alarming three or four times the rate of the general public - roughly equal to the rate of male doctors.

13 'Suicide among doctors. :789.

14 Franklin RA. One hundred doctors at the retreat: A contribution to the subject of mental disorder in the medical profession. British Journal of Psychiatry. 1977;131:11-14. on 12. [PubMed: 884407]

15 Franklin. One hundred doctors at the retreat. :13. [PubMed: 884407]

16 a'Brook. Psychiatric illness in the medical profession. :1020.

17 a'Brook. Psychiatric illness in the medical profession. :1018.

18 Murray Robin M. Characteristics and prognosis of alcoholic doctors. British Medical Journal. 1976 December 15;:1537-1559. on 1538. [PMC free article: PMC1690154] [PubMed: 1009387]

19 Glatt MM. letter to the British Medical Journal. 1979 September 22;:732. [PMC free article: PMC1596271] [PubMed: 509081]

20 Short SED. Psychiatric illness in physicians. Journal of the Canadian Medical Association. 1979;121:283-288. on 287.

21 Short. Psychiatric illness in physicians. :287.

22 Short. Psychiatric illness in physicians. :287.

23 Leading article. Doctors’ diseases. British Medical Journal. 1967 December 9;:467-468. on 467.

24 See for example the account from Murray Robin M. The alcoholic doctor. British Journal of Hospital Medicine. 1977;18:144-149. on 147. [PubMed: 890192]

25 Lloyd Gareth. personal paper 'I am an alcoholic. British Medical Journal. 1982 September 18;:785-786. [PMC free article: PMC1499524] [PubMed: 6811002] (MRCOG - Membership of the Royal College of Obstetricians and Gyneacologists examination.)

26 Lloyd. I am an alcoholic. :786.

27 Glatt. Alcoholism, an occupational hazard for doctors. :88.

28 a'Brook. Psychiatric illness in the medical profession. :1018.

29 Murray. The alcoholic doctor. :148.

30 Duffy John C, Litin Edward M. Psychiatric morbidity of physicians. Journal of the American Medical Association. 1964;189:989-992. on 990. [PubMed: 14188887]

31 a'Brook. Psychiatric illness in the medical profession. :1017.

32 Duffy, Litin Psychiatric morbidity of physicians. :99. 
33 Allibone A. The health of doctors. In: Pereira Gray DJ, editor. The Medical Annual. Bristol: Wright; 1983. pp. 141-150. on p. 146.

34 Allibone. The health of doctors. 147:146.

35 Allibone. The health of doctors. :141.

36 Simmonds AL. letter to the British Medical Journal. 1983 December 10;:1796.

37 Allibone A. letter to the British Medical Journal. 1983 December 10;:1796.

38 Samanta Ash, Samanta Jo. Regulation of the medical profession: Fantasy, reality and legality. Journal of the Royal Society of Medicine. 2004;97:211-218. on 211. [PMC free article: PMC1079459] [PubMed: 15121809]

39 Notes and news. Alcoholism among the medical profession. Lancet. 1978 December 2;:1215.

40 Allibone. The health of doctors. :142.

41 Notes and News. Alcoholism among the medical profession. :1215.

42 Marien B, Mckinna F. Editorial. The elephant in the room. Clinical Oncology. 2012;24:654-656. on 654. [PubMed: 22906545]

43 a'Brook, et al. Psychiatric illness in the medical profession. 1013:1017.

44 Glatt. Alcoholism, an occupational hazard for doctors. :87.

45 Murray. The alcoholic doctor. :149.

46 Glatt. Alcoholism, an occupational hazard for doctors. :87.

47 Interview with David Palmer.

48 Interview with Christian Edwards.

49 Evans JL. Psychiatric illness in the physician's wife. American Journal of Psychiatry. 1965;122:159-163. [PubMed: 14313429], cited in a'Brook Psychiatric illness in the medical profession', 1018.

50 Evans. Psychiatric illness in the physician's wife', cited in a'Brook, Psychiatric illness in the medical profession. :1018.

51 Evans. Psychiatric illness in the physician's wife', cited in Leading Article, Doctors' diseases. British Medical Journal. 1967 December $9 ;: 567-568$. on 568 .

52 S. E. D. Short, Review Article. Psychiatric illness in physicians. Journal of the Canadian Medical Association. 1979;121:238-288. on 284.

53 Short. Psychiatric illness in physicians. :284.

54 Duffy. Psychiatric morbidity of physicians. :98.

55 Jill Pereira Gray, Editorial. The family doctor's family. Journal of the Royal College of General Practitioners. 1978;28:579.

56 Allibone. The health of doctors. :144.

57 Glatt. Alcoholism, an occupational hazard for doctors. :90.

58 Glatt. Alcoholism, an occupational hazard for doctors. :90.

59 Glatt. letter to the British Medical Journal. 1979 September 22;:732.

60 a'Brook, et al. Psychiatric illness in the medical profession. :1021.

61 See http://www.bddg.org/10/history-of-bddg/ accessed on 15 January 2015. Nurses and female doctors have increasingly been vulnerable to mental illness, addiction and suicide.

62 Glatt. letter to the British Medical Journal. 1979 September 22;:732.

63 Allibone. The health of doctors. :145.

64 Allibone. The health of doctors. :149.

65 Short. Psychiatric illness in physicians. :287.

66 Corson Samuel A, Corson Elizabeth O’Leary. Working situations and psychophysiological pathology - a systems approach. In: Levi 
Lennart, editor. Society, Stress and Disease. Volume 4. Oxford: Oxford University Press; 1981. pp. 226-247.pp. 236-237. Working Life.

67 Corson, Corson Working situations. :237.

68 Corson, Corson Working situations. :237.

69 Corson, Corson Working situations. :238.

70 Corson, Corson Working situations. :238-239.

71 Panayi Panikos. An Immigration History of Britain: Multicultural Racism since 1800. Harlow: Pearso; 2010. p. 23.

72 Panayi. An Immigration History of Britain. :24.

73 Panayi. An Immigration History of Britain. :24.

74 Panayi. An Immigration History of Britain. :25.

75 Baker CC, Pocock SJ. Ethnic differences in certified sickness absence. British Journal of Industrial Medicine. 1982;39:277-282. on 277. [PMC free article: PMC1009024] [PubMed: 7093156]

76 Zwingmann Charles, Pfister-Ammende Maria. Uprooting and After. New York: Springer Verlag; 1973. pp. 2-3. Studies prior to this were confined to lunatic asylums in the US during the nineteenth century.

77 Littlewood Roland, Lipsedge Maurice. Aliens and Alienists: Ethnic Minorities and Psychiatry. New York and Hove: Routledge; 1997. edition) p. xi. Original emphasis.

78 Notable studies include: Malzberg B. Mental disease in New York State according to nativity and parentage. Mental Hygiene. 19:635660. and Ødegaard Ø. Acta Psychiatrica et Neurologica. Copenhagen: Levin and Munksgard; 1935. Emigration and insanity: A study of mental disease among the Norwegian-born population of Minnesota.

79 Bains Jatinder. Race, culture and psychiatry: A history of transcultural psychiatry. History of Psychiatry. 2005;16(2):139-154. [PubMed: 16013117], on 139. Theories about the association between the 'civilising process' and insanity have a much longer history dating back to the nineteenth century, leading subsequently to late nineteenth-century ideas about the 'inferior' intelligence of primitive people within the context of social Darwinism. See Raimundo Oda Ana Maria G, et al. Some origins of cross-cultural psychiatry. History of Psychiatry. 2005;16(2):155-169. [PubMed: 16013118]

80 Bains. Race, culture and psychiatry. :141.

81 Bains. Race, culture and psychiatry. :145.

82 Dein Simon, Bhui Kamaldeep Singh. The crossroads of anthropology and epidemiology: Current research in cultural psychiatry in the UK. Transcultural Psychiatry. 2013;50(6):769-791. on 771. [PMC free article: PMC4107756] [PubMed: 24114263]

83 Dein, Bhui The crossroads of anthropology and epidemiology. :771. [PMC free article: PMC4107756] [PubMed: 24114263]

84 Dein, Bhui The crossroads of anthropology and epidemiology. :771. [PMC free article: PMC4107756] [PubMed: 24114263]

85 Mezey AG. Psychiatric aspects of human migrations. International Journal of Social Psychiatry. 1960;5:245-260. on 245.

86 Ødegaard. cited in Mezey Psychiatric aspects of human migration. :248.

87 Mezey. Psychiatric aspects of human migrants. :258.

88 Mezey. Psychiatric aspects of human migrants. :252.

89 Murphy HBM. Migration and the major mental disorders: A reappraisal. In: Zwingmann, Pfister-Ammende, editors. Uprooting and After. New York: Springer Verlag; 1973. pp. 204-220. on p. 207.

90 Murphy. Migration and the major mental disorders. :209.

91 Murphy. Migration and the major mental disorders. :210.

92 Mezey. Psychiatric aspects of human migrants. :252.

93 Littlewood, Lipsedge Aliens and Alienists. :128.

94 Littlewood, Lipsedge Aliens and Alienists. :128. 
95 Smith DJ. Racial Disadvantage in Britain. Harmondsworth: Penguin; 1977. ), cited in Littlewood and Lipsedge. Aliens and Alienists. :129.

96 Early international studies include: Collins CP. Sickness absence in the three principal ethnic divisions of Singapore. British Journal of Industrial Medicine. 1962;19:116-122. [PMC free article: PMC1038168] [PubMed: 13880579] and WHO. Health Aspects of Labour Migration: Report on a Working Group Convened by the Regional Office for Europe of the WHO, Algiers, 1973. Copenhagen: WHO; 1974.

97 Baker, Pocock Ethnic differences in certified sickness absence. :277. [PMC free article: PMC1009024] [PubMed: 7093156]

98 Baker, Pocock Ethnic differences in certified sickness absence. :279. [PMC free article: PMC1009024] [PubMed: 7093156]

99 Baker, Pocock Ethnic differences in certified sickness absence. :281. [PMC free article: PMC1009024] [PubMed: 7093156]

100 Crane Stuart. Problems of an immigrant population', Section of General Practice. Proceedings of the Royal Society of Medicine. 1970;63:629-631. on 629. [PMC free article: PMC1811573] [PubMed: 5453473]

101 Crane. Problems of an immigrant population. :630.

102 Crane. Problems of an immigrant population. :631.

103 Hashmi Farrukh. Immigrants and emotional stress', Section of General Practice. Proceedings of the Royal Society of Medicine. 1970;63:631-632. on 631. [PMC free article: PMC1811578] [PubMed: 5453474]

104 Hashmi. Immigrants and emotional stress. :632. [PMC free article: PMC1811578] [PubMed: 5453474]

105 Hashmi. Immigrants and emotional stress. :632. [PMC free article: PMC1811578] [PubMed: 5453474]

106 Carne. Problems of an immigrant community. :629.

107 Carne. Problems of an immigrant community. :629.

108 Interview with James Robertson.

109 Interview with James Robertson.

110 Interview with James Robertson.

111 Interview with Sarah Hall.

112 Interview with James Robertson.

113 A point made in both interviews with Robertson and Hall.

114 Interview with Sarah Hall. James Robertson also noted that somatisation was more common in immigrant groups.

115 Interview with James Robertson.

116 Interview with Sarah Hall.

117 Interview with Sarah Hall.

118 A jinn is a supernatural spirit in Islamic mythology.

119 Interview with Sarah Hall.

120 Carne. Problems of an immigrant population. :636.

121 Carne. Problems of an immigrant population. :636.

122 Katon Wayne, Kleinman Arthur, Rosen Gary. Depression and somatisation: A review', part 1. The American Journal of Medicine. 1982;72:127-135. on 131. [PubMed: 7058818]

123 A term used by Kleinman in 'Depression, somatization and the "new crosscultural psychiatry" :4.

124 Kleinman. Depression, somatization and the "new cross-cultural psychiatry" :4.

125 Department of Health. Invisible Patients: Report of the Working Group on the Health of Health Professionals. 2010:7.

126 Department of Health. Invisible Patients. :36.

127 Department of Health. Invisible Patients. :34. It should be noted that women doctors are now particularly vulnerable to suicide. 
128 Department of Health. Invisible Patients. 34:45-47.

129 See Lang Susan S. Economists coin new word, "presenteeism," to describe worker slowdowns that account for up to 60 percent of employer health costs. Cornell Chronicle. 2004 April 20; [accessed 9 March 2015]; at http://www.news.cornell.edu/stories/2004 /04/new-word-job-health-problem-presenteeism,

130 Department of Health. Invisible Patients. :17.

131 The Peninsula Medical School in south west England was highlighted in this respect. Department of Health. Invisible Patients. :35.

132 Mental Health Foundation website. Black and minority ethnic communities. [accessed 13 March 2015]; at http://www.mentalhealth .org.uk/help-information/mental-health-a-z/B/BME-communities/

133 See, for example, Greene Ruby, Pugh Richard, Roberts Diane. Black and Minority Ethnic Parents with Mental Health Problems and their Children. London: Social Care Institute for Excellence; 2008. p. 1. Research Briefing.

134 Greene, Pugh, Roberts Black and Minority Ethnic Parents. :3.

135 Rehman Hamid, Owen David. Mental Health Survey of Ethnic Minorities. Ethnos Research and Consultancy; 2013.

136 McKenzie Kwame. Improving mental healthcare for ethnic minorities. Advances in Psychiatric Treatment. 2008;14:285-291. on 287.

\section{(C) Ali Haggett 2015 .}

The author has asserted his right to be identified as the author of this work in accordance with the Copyright, Designs and Patents Act 1988.

Palgrave Macmillan in the UK is an imprint of Macmillan Publishers Limited, registered in England, company number 785998, of Houndmills, Basingstoke, Hampshire RG21 6XS.

Palgrave Macmillan in the US is a division of St Martin's Press LLC, 175 Fifth Avenue, New York, NY 10010.

Palgrave Macmillan is the global academic imprint of the above companies and has companies and representatives throughout the world.

Palgrave ${ }^{\circledR}$ and Macmillan ${ }^{\circledR}$ are registered trademarks in the United States, the United Kingdom, Europe and other countries.

Except where otherwise noted, this work is licensed under a Creative Commons Attribution 3.o Unported License. To view a copy of this license, visit http://creativecommons.org/licenses/by/3.0/

Monographs, or book chapters, which are outputs of Wellcome Trust funding have been made freely available as part of the Wellcome Trust's open access policy

Bookshelf ID: NBK333537 